\title{
EchoGéo
}

6 | 2008

La Guyane

\section{La Guyane, un territoire Caraïbe en voie de sud- américanisation}

\section{Stéphane Granger}

\section{OpenEdition}

1 Journals

Édition électronique

URL : https://journals.openedition.org/echogeo/6503

DOI : 10.4000/echogeo.6503

ISSN : 1963-1197

\section{Éditeur}

Pôle de recherche pour l'organisation et la diffusion de l'information géographique (CNRS UMR 8586)

Référence électronique

Stéphane Granger, « La Guyane, un territoire Caraïbe en voie de sud-américanisation », EchoGéo [En ligne], 6 | 2008, mis en ligne le 09 juillet 2008, consulté le 31 juillet 2021. URL : http:// journals.openedition.org/echogeo/6503; DOI : https://doi.org/10.4000/echogeo.6503

Ce document a été généré automatiquement le 31 juillet 2021.

EchoGéo est mis à disposition selon les termes de la licence Creative Commons Attribution - Pas d'Utilisation Commerciale - Pas de Modification 4.0 International (CC BY-NC-ND) 


\title{
La Guyane, un territoire Caraibe en voie de sud-américanisation
}

\author{
Stéphane Granger
}

1 En juillet 2006, plusieurs lycéens guyanais descendaient l'Amazone avec d'autres jeunes de tous les pays signataires du Traité de Coopération amazonien, dans le cadre d'un projet scientifique et culturel international qui pour la première fois intégrait la Guyane française. Celle-ci était enfin reconnue par ses voisins comme un territoire amazonien à part entière après des années, sinon des siècles d'ostracisme. En effet, dernière portion non-souveraine de l'Amérique du sud, elle connaît une étrange situation juridico-politique : tout en étant géographiquement en Amérique du sud, elle est considérée historiquement et culturellement comme plutôt caraïbe, alors qu'elle est en même temps un département et une Région de la République française, et donc de l'Union Européenne.

2 Longtemps la Guyane a tourné le dos pour des raisons historiques et politiques au reste du sous-continent sud-américain, et celui-ci la marginalisa longtemps à cause de sa situation de région européenne. Mais aujourd'hui cette même situation la rend attractive pour les autres pays de la région. Si les autorités politiques et économiques commencent tout juste à découvrir les possibilités de cette étonnante situation à la croisée de l'Union Européenne, du Caricom ${ }^{1}$ et du Mercosud, c'est pourtant par des activités illicites que la Guyane a d'abord commencé à s'intégrer dans son environnement. Mais elle se heurte encore aux grands déséquilibres régionaux et aux réalités géopolitiques et économiques.

\section{Une « Caraïbe européenne »}

3 Située sur la côte septentrionale de l'Amérique du sud, la Guyane française tout comme le Surinam et le Guyana est un territoire plus caraïbe que sud-américain par son histoire et sa culture : séparée du reste du sous-continent par la forêt amazonienne et son statut européen, peuplée essentiellement sur sa façade atlantique, pratiquant des langues, français et créole, parlées aux Antilles et en Europe mais pas ailleurs en 
Amérique du sud, elle appartient incontestablement à l'aire caraïbe dont elle constitua comme ses voisines une extension protectrice pour les puissances coloniales d'Europe de l'ouest. Elle représente par ailleurs la France à l'AEC ${ }^{2}$, avec la Martinique et la Guadeloupe, et accueillit en 2005 la conférence internationale pour régler le problème d'Haïti.

4 Les Guyanes avaient en fait été attribuées aux Espagnols par le traité de Tordesillas en 1494, mais ceux-ci les négligèrent, les jugeant moins intéressantes et hospitalières que le Pérou ou le Mexique. Aussi, Français, Hollandais et Britanniques profitèrent de leur absence pour prendre pied sur le sous-continent sud-américain, mais ils furent rapidement limités par la forêt amazonienne et la présence des puissances ibériques, détentrices « légitimes » du Nouveau Monde.

Depuis le XVII ${ }^{e}$ siècle la présence des trois petites Guyanes constitue ainsi une anomalie au sein de ce sous-continent auquel elles tournent le dos, d'autant plus que, non concernées par le mouvement d'indépendance des colonies espagnoles et portugaises de la première moitié $\mathrm{du} \mathrm{XIX}^{\mathrm{e}}$ siècle, elles ne quittèrent que récemment le giron européen. La Grande-Bretagne accordait en 1966 l'indépendance à la Guyane britannique devenue Guyana, les Pays-Bas faisaient de même avec la Guyane hollandaise, devenue Surinam en 1975. La Guyane française au contraire renforçait son intégration à la métropole en devenant département d'outre-mer de la République en 1946, et, depuis l'indépendance du Belize en 1981, elle constitue le dernier territoire continental américain relevant d'une puissance européenne, statut qu'elle partage avec quelques îles de la Caraïbe encore possessions néerlandaises, britanniques ou françaises.

6 Une fois indépendantes, les Guyanes conservèrent leurs liens privilégiés avec l'ancienne métropole, leurs sœurs antillaises et le monde caraïbe au détriment de relations avec le reste de l'Amérique du sud. Guyana et Surinam furent certes admis, à l'initiative d'un Brésil en mal de bons voisinages, au Traité de Coopération Amazonien en 1978, avec tous les autres pays d'écosystème amazonien mais à l'exception de la Guyane française, du fait de sa «situation coloniale» qui aurait impliqué la participation de la France, puissance européenne. Préférant l'orientation caraïbe, ils intégraient cependant les pays $\mathrm{ACP}^{3}$, institués d'ailleurs à Georgetown, capitale du Guyana, en 1975, puis l'Association des Etats de la Caraïbe en 1994. Enfin, en 1995, le Caricom, dont le siège est aussi à Georgetown et où le Surinam constitue, avec Haïti, le seul membre non issu du Commonwealth.

7 Exclue de fait des échanges et des réseaux économiques régionaux et isolée au sein de son environnement, la Guyane française se voyait intégrée à l'Union Européenne en tant que département français : ce statut ainsi que les coûts élevés de salaires et de production qui en découlent font qu'à l'exception des hydrocarbures en provenance de Trinidad l'essentiel de ses échanges économiques se fait avec la France hexagonale, l'Union Européenne et les Antilles françaises. La Guyane est du coup relativement isolée du reste du continent: seulement une route vers le Surinam accessible par bac, et une route toute récente vers le Brésil, achevée en 2003, mais l'on ne peut ensuite traverser le fleuve-frontière Oyapock qu'au moyen d'une barge onéreuse et non homologuée. 


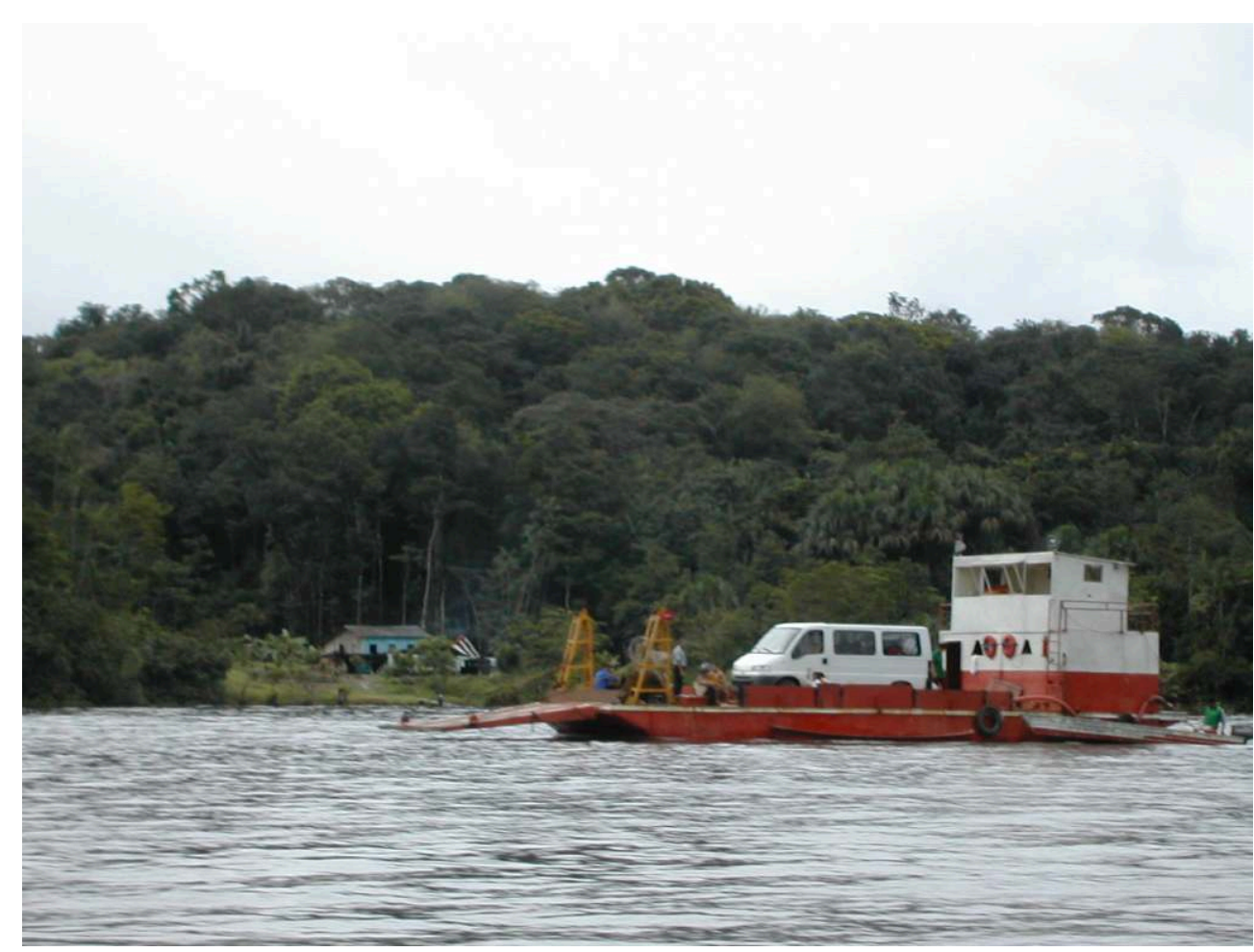

8 Les liaisons aériennes ne sont guère plus développées : celle vers le Surinam n'est pour l'instant plus assurée, et la liaison avec Belém au Brésil ne s'effectue plus que trois fois par semaine, alors qu'elle était autrefois quotidienne.

Croquis 1- Liaisons routière et aériennes depuis la Guyane

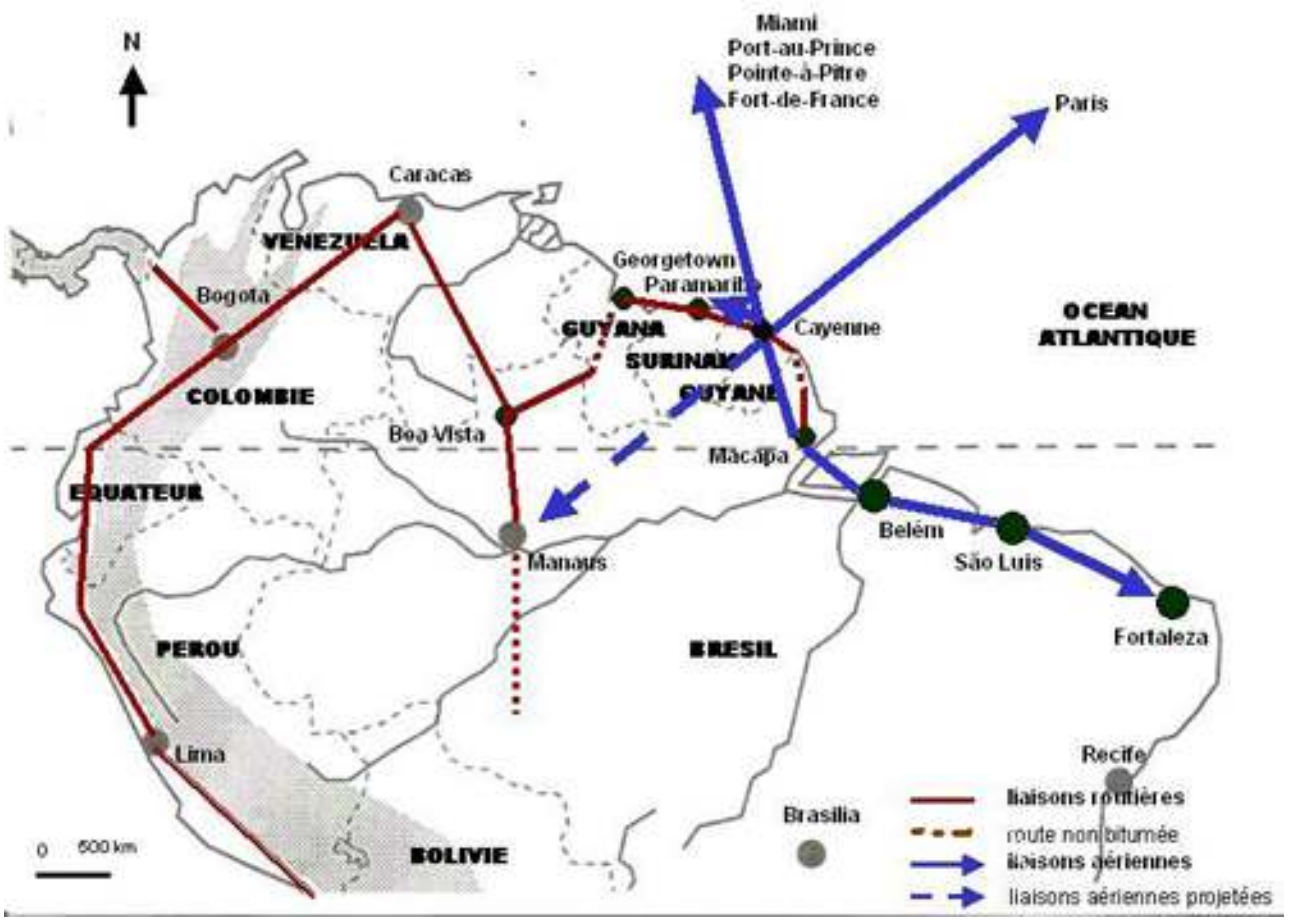

Auteur: S. Granger. 
La Guyane est ainsi une région européenne en Amérique du sud: "port spatial de l'Europe » grâce à la présence du Centre Spatial Guyanais à Kourou, elle connaît une situation bien plus favorable que les pays voisins grâce aux transferts et à la redistribution nationale, mais la forte fécondité et la mortalité infantile relativement élevée rappellent davantage l'Amérique du sud que la France et l'Europe. Aussi bénéficie-t-elle des fonds structurels européens au titre des régions européennes les plus pauvres, mais également en tant que "région ultrapériphérique » de l'Union Européenne, statut qu'elle partage avec les autres départements français d'outre-mer, les îles portugaises de Madère et des Açores, et l'archipel espagnol des Canaries.

10 Mais ces aides, qui font des départements français d'Amérique les territoires les plus riches de la Caraïbe et de l'Amérique du sud, entraînent un effet pervers, en aggravant le côté artificiel de la prospérité guyanaise tout en accentuant l'écart d'équipement et de niveau de vie avec les pays voisins : coûts, prix et salaires sont quatre à dix fois supérieurs à ceux du Brésil et du Surinam. Or c'est paradoxalement ce haut niveau de vie dû à l'appartenance européenne qui permet depuis quelques années une insertion progressive dans le sous-continent.

\section{Une pression migratoire caraïbe et amazonienne}

11 Cette île européenne que constitue la Guyane au milieu d'un océan de pauvreté attire de nombreux immigrants issus des pays proches. Aux quelques centaines de Colombiens et surtout de Brésiliens (ces derniers constituant désormais près de $10 \%$ de la population) venus au dans les années 1960 pour la construction de la base spatiale, se sont ajoutés environ 10000 Surinamiens fuyant la guerre civile au début des années 1990, puis, à ce flot continu, des milliers d'Haïtiens (désormais la communauté la plus nombreuse), Dominicains, Guyaniens... La population étrangère en Guyane constituerait près de $40 \%$ de la population totale, certaines estimations la voient même majoritaire, et plus de la moitié des naissances lui sont imputées car les comportements démographiques restent les mêmes que dans le pays d'origine, où la fécondité est très élevée. 
Evolution des structures administratives de la Guyane française depuis 1946 en Guyane et localisation des communautés locales depuis le début du XXle siècle

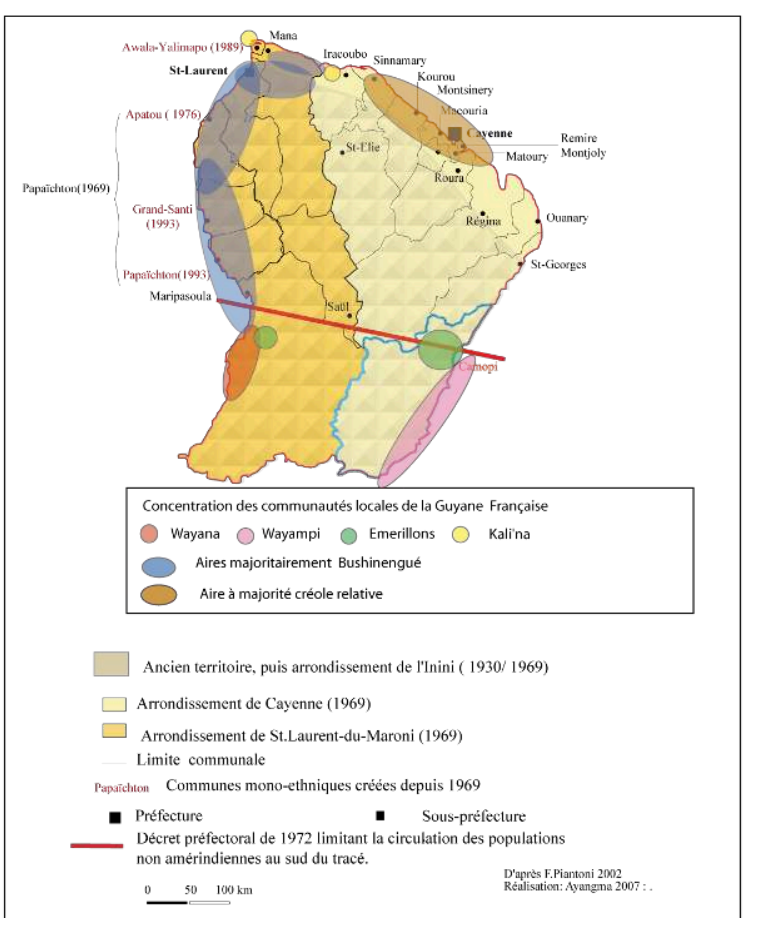

12 Ces immigrants, par la facilité avec laquelle ils parviennent en Guyane, passant par le Surinam ou le Brésil, montrent à ses habitants qu'elle appartient bien à ce continent. La Guyane, par la porosité de ses frontières et le marché qu'elle constitue, est également une plaque tournante pour la drogue venue de Colombie via le Surinam, et son immensité non maîtrisée attise la convoitise de milliers de garimpeiros (chercheurs d'or) brésiliens dans le sud du territoire, où ils créent de véritables agglomérations équipées en bars et magasins...

13 Ce phénomène se retrouve d'ailleurs dans toute l'Amazonie septentrionale : Surinam, Guyana, Venezuela et Colombie, dont la souveraineté ainsi remise en cause montre bien l'aspect virtuel des frontières politiques. On va jusqu'à évoquer une délinquance de type sud-américain en Guyane, où les délits commis par des immigrants clandestins rappellent par leur nature et leur violence ceux du Brésil ou de la Colombie.

En outre, symbole de la « continentalisation » croissante de ces migrations, la Guyane connaît depuis une dizaine d'années une irruption assez importante de Péruviens qui seraient maintenant un bon millier, tous passés par Iquitos, Manaus, puis l'Amapá et maintenant le Surinam. 
Croquis 2 - Les activités illicites en Guyane et en Amazonie

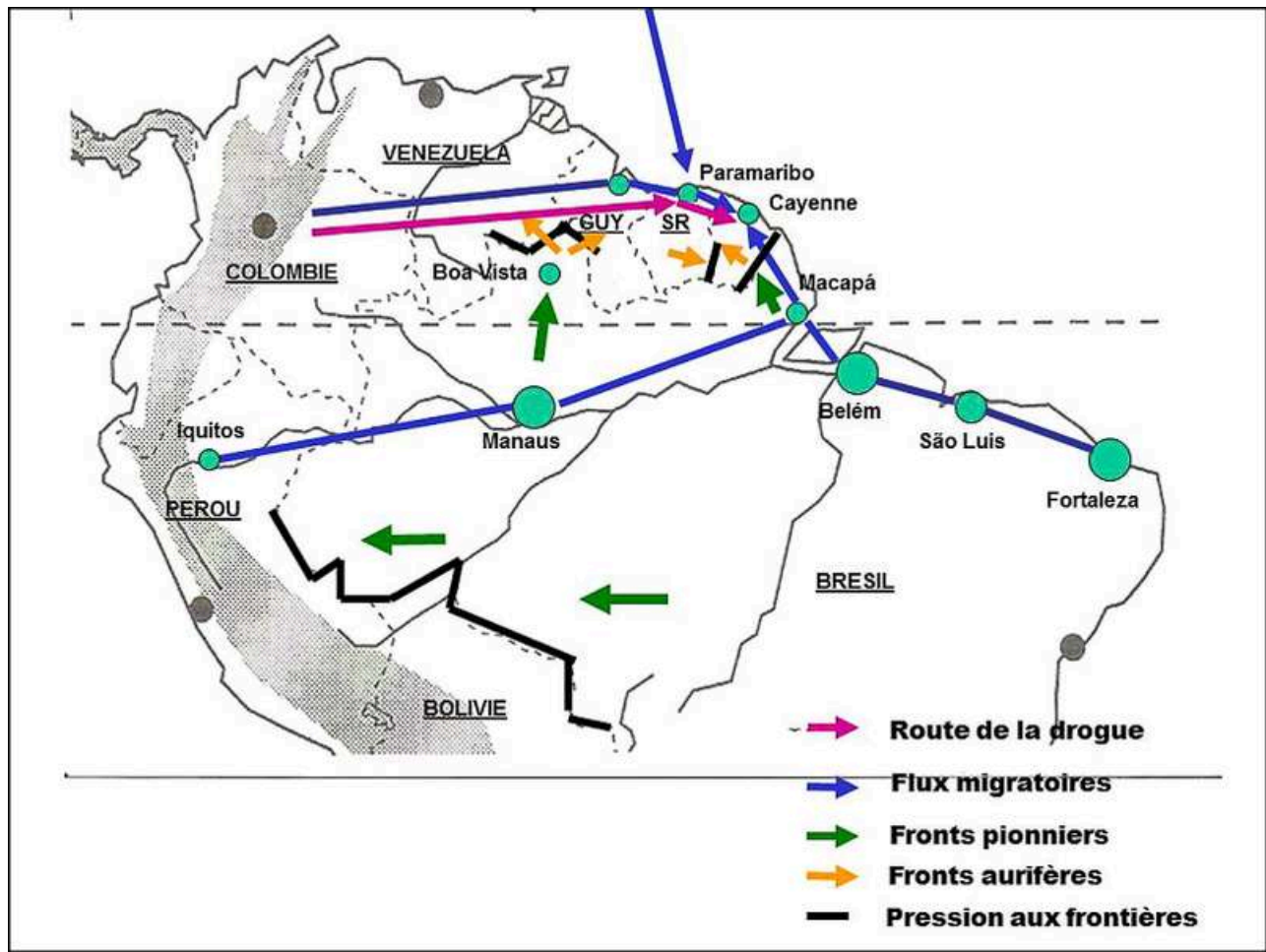

Auteur: S. Granger.

15 Le Surinam est en effet la plaque tournante des migrations caraïbes (haïtiennes et dominicaines), mais aussi sud-américaines vers la Guyane : sachant qu'il n'est qu'un pays de transit vers la Guyane française, il est moins exigeant pour les visas, ou moins regardant. Tout comme le reste de l'Amazonie septentrionale, la Guyane se retrouve ainsi comme un prolongement imprévu des fronts pionniers ou aurifères brésiliens.

Tableau 1 - les reconduites à la frontière en 1996 et 2006

\begin{tabular}{|l|l|l|}
\hline & 1996 & 2006 \\
\hline Haïtiens & 598 & 190 \\
\hline Dominicains & 108 & 107 \\
\hline $\begin{array}{l}\text { Divers Caraïbes } \\
\text { (Ste Lucie, Barbade, } \\
\text { Jamaïque, Trinidad) }\end{array}$ & 18 & 0 \\
\hline Guyaniens & 380 & 138 \\
\hline Surinamiens & 3571 & 3593 \\
\hline Brésiliens & 351 & 4036 \\
\hline Vénézuéliens & 2 & 0 \\
\hline
\end{tabular}




\begin{tabular}{|l|l|l|}
\hline Colombiens & 5 & 1 \\
\hline Péruviens & 11 & 34 \\
\hline Boliviens & 0 & 4 \\
\hline Uruguayens & 0 & 1 \\
\hline Argentins & 1 & 2 \\
\hline
\end{tabular}

Source : DDCILEC Guyane.

Le tableau des reconduites à la frontière en 2006 ci-dessus montre le poids croissant de certaines migrations frontalières et continentales. La forte augmentation pour le Brésil s'explique par le récent achèvement de la route de Cayenne vers le Brésil, qui fonctionne un peu comme un appel d'air, mais sur laquelle les candidats à l'immigration se font aussi attraper plus facilement. L'évolution des autres nationalités est aussi largement tributaire des possibilités et conditions juridiques de rapatriement des clandestins.

17 Finalement, c'est d'abord par des activités illicites (drogue, contrebande, orpaillage et immigration clandestine) que la Guyane s'est intégrée au sous-continent. Les responsables politiques locaux, grâce aux évolutions statutaires leur donnant plus d'initiative (lois de Décentralisation en France, nouvelle constitution au Brésil...) ont alors suivi le mouvement à partir des années 1990 et cherché à se rapprocher, les uns dans l'espoir qu'une politique de coopération avec les pays voisins puisse atténuer les flux migratoires vers la Guyane, les autres que des accords permettent un désenclavement et l'accès à de nouveaux marchés.

\section{La difficile insertion d'une région européenne en Amérique du sud}

Les accords de coopération frontaliers les plus poussés sont depuis 1996 avec l'Etat brésilien d'Amapá et ont permis une multiplication des échanges surtout scientifiques, culturels, scolaires et sportifs. Cette coopération fut impulsée au plus haut niveau par les présidents français Jacques Chirac et brésilien Fernando Henrique Cardoso, qui se sont rencontrés en Guyane en 1997, et y ont décidé la construction d'un pont sur le fleuve frontalier Oyapock. Financé par le Brésil, la France et l'Union Européenne, il symbolisera cette insertion de la Guyane en Amérique du sud tout en permettant une ouverture du nord du Brésil vers la Caraïbe. D'autre part de nouveaux projets de liaisons aériennes voient le jour : Air France remettra très prochainement en service la ligne Cayenne-Paramaribo, alors que la ligne Cayenne-Belém est désormais prolongée vers Fortaleza et Recife en attendant Salvador, même si la fréquence des avions a été réduite.

Enfin, une compagnie brésilienne cherche depuis l'année dernière à créer et exploiter une ligne Cayenne-Manaus, qui relierait la capitale guyanaise au cœur de l'Amazonie et permettrait des correspondances directes avec Panamá et les pays andins. Il est à noter que Manaus est une destination très prisée de touristes originaires d'Allemagne et 
d'Europe du nord férus de tourisme écologique ; avec cette ligne Cayenne deviendrait la porte d'entrée de l'Amérique du sud pour l'Europe et constituerait ainsi une véritable plaque tournante entre ces deux continents.

Cette intégration croissante de la Guyane dans son continent d'origine est en grande partie due au Brésil, qui a compris, peut-être plus que la France, l'intérêt et l'originalité de cette région pour sa stratégie d'affirmation régionale et continentale. En effet, même si la Guyane constitue un faible marché avec ses 200000 habitants, c'est parce qu'elle est la "porte d'entrée » de l'Union Européenne qu'il tient autant à ce projet de pont qui permettra une liaison routière entre Guyane et Etat d'Amapá, c'est à dire entre France et Brésil (avec $730 \mathrm{~km}$ la frontière guyano-brésilienne est la plus grande frontière terrestre de France), mais aussi entre Union Européenne et Mercosud... tout en désenclavant ses Etats du nord entre lesquels s'insèrent les Guyanes : l'Amapá est le seul Etat brésilien non encore relié par voie terrestre au reste du pays.

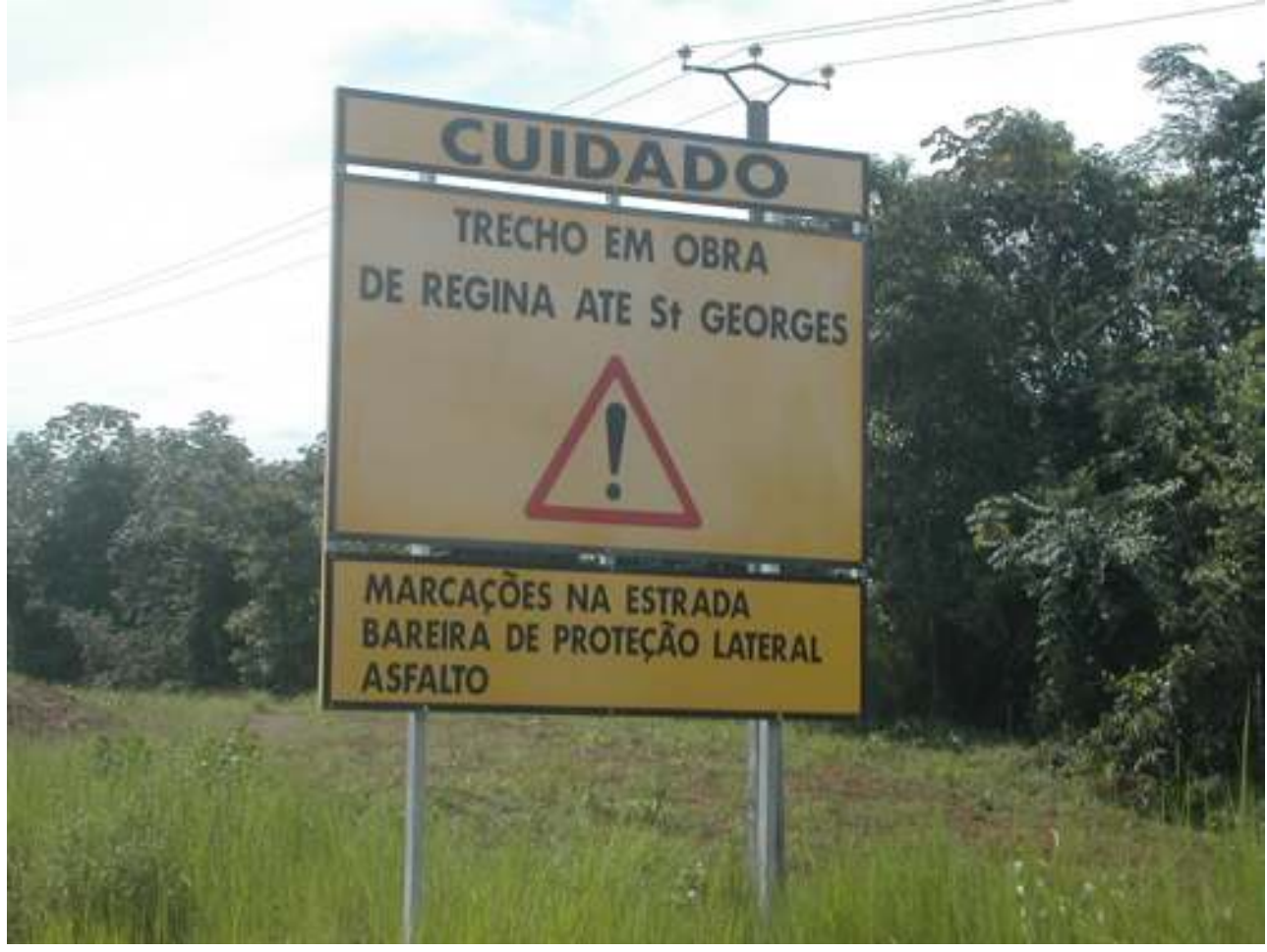

21 Aussi, désormais mieux connue des Brésiliens du nord, la Guyane est chaque année invitée en tant que telle dans des manifestations régionales comme le salon du livre amazonien de Belém, ou la foire industrielle de Manaus, alors que les responsables politiques guyanais cherchent à étendre leurs relations avec d'autres Etats brésiliens plus dynamiques et prometteurs (Amazonas, Pará, Ceará).

Car le paradoxe veut que ce soit l'appartenance européenne de la Guyane française qui la rende attractive pour les autres pays de la région, après l'en avoir éloignée. C'est ce qu'a montré en 2004 le désir des pays signataires du Traité de Coopération Amazonien de l'associer en tant que membre observateur à travers la France. L'OTCA avait pourtant au début exclu la Guyane comme possession européenne, elle fut en effet longtemps ostracisée par les pays du continent comme « anomalie coloniale ». Mais les temps changent et désormais son partenariat est recherché: par des réponses scientifiques "européennes" (Institut Pasteur, IRD, CIRAD...) à des problèmes communs comme la lutte contre certains parasites, avec des budgets et des 
compétences que l'on ne trouve pas forcément dans les autres pays amazoniens, elle a des expériences à faire partager à ses voisins. Ceux-ci espèrent aussi se rapprocher de l'Europe pour échapper à l'emprise des Etats-Unis, et ils comptent sur la Guyane malgré sa situation marginale pour en constituer une porte d'entrée. De plus, par sa situation géographique elle ne pouvait plus rester à l'écart des projets d'harmonisation des politiques amazoniennes, qu'elles soient environnementales ou même policières, dans un continent en plein processus d'intégration, où les problèmes dépassent des frontières nationales souvent artificielles. Mais c'est aussi en raison de ce statut européen qu'elle ne pourra prendre part aux votes.

Cependant, les limites statutaires font que pour les négociations les pays de l'OTCA passent directement par la France. En contournant ainsi la Guyane, ils montrent qu'elle n'est encore pour eux qu'une excroissance de la France en terre sud-américaine et non un territoire à la personnalité propre.

Enfin, la Communauté sud-américaine des Nations a exclu en décembre dernier la Guyane française et elle seule des dispositions visant à la suppression du passeport et du visa entre les pays du sous-continent, car la réciprocité eût été impossible en raison des intenses flux migratoires et de la nationalité française des Guyanais. Le processus d'intégration sud-américaine de la Guyane a trouvé là sa limite, du fait même de ce qui avait au début permis de l'amorcer : son appartenance française et européenne. Et ceci alors que la coopération économique n'a pas eu les effets escomptés, peu de produits franchissent légalement la frontière, les produits agro-alimentaires consommés en Guyane viennent encore pour la plupart de l'Europe, y compris les jus de fruits tropicaux! La coopération n'a pas vaincu les logiques protectionnistes des grands ensembles économiques qui la coupent de son environnement géographique. Et il sera toujours plus facile à un Guyanais, comme n'importe quel citoyen européen, de résider et travailler en Allemagne ou en Italie qu'au Brésil ou au Surinam, et plus aisé et moins onéreux pour un Sud-Américain de se rendre à Paris qu'à Cayenne.. C'est le contrecoup de l'intégration européenne, et de la faiblesse des communications entre la Guyane et les autres pays de la région qui en découle.

La Guyane est ainsi à la croisée de plusieurs chemins et d'ensembles politicoéconomiques: Union Européenne, AEC, Mercosud, Caricom, OTCA, CSAN. Les immigrants auront été les pionniers d'une intégration progressive mais limitée de la Guyane dans son environnement amazonien et donc sud-américain, en suscitant une prise de conscience des avantages d'une région européenne en Amérique du sud. Dans un monde de plus en plus globalisé, ces appartenances multiples devraient constituer un atout non négligeable pour elle comme pour l'Europe et l'Amérique du sud. Terre longtemps exclue et terre d'exclusion avec le Bagne, elle pourrait devenir une interface capitale pour une Union Européenne dans laquelle son poids va décroître en raison des élargissements successifs qui réduisent l'assiette des fonds structurels. Mais en dépit de la fameuse boutade du président brésilien Lula, pour qui la France fera bientôt partie du Mercosud grâce au pont sur l'Oyapock, la Guyane, à cause de son statut et de sa richesse relative, restera pour longtemps encore une région européenne en Amérique du sud plutôt qu'un véritable territoire sud-américain. 


\section{BIBLIOGRAPHIE}

Bataillon Claude, Deler Jean-Paul, Théry Hervé, 1991, Géographie universelle tome 3 : Amérique latine, Belin, collection Reclus.

Blanquet Jean-Michel, 2005, « Les Guyanes et les Amériques entre continentalisation et « océanisation », Etudes de la documentation française, Amérique latine, p. 69-79.

Calmont André, 2006, « Immigration et développement en Guyane depuis la départementalisation », in Mam-Lam-Fouck Serge (coord.), L'histoire de la Guyane depuis les civilisations amérindiennes, Ibis Rouge Editions, p. 427-444.

Granger Stéphane, 2001, « Coopération régionale », in Barret Jacques (coord.), Atlas illustré de la Guyane, IRD, p. 98-99.

- 2006, « La Guyane, une région européenne en Amérique du sud », Actes du Festival international de géographie de Saint-Dié, www.fig-st-die.education.fr/actes/actes_2006

- 2007, « Guyane et Surinam à l'intersection des migrations caraïbes et sud-américaines », Terres d'Amériques n 6, «Dynamiques migratoires de la Caraïbe », Karthala, p. 287-301.

INSEE, 2004, Panorama de l'espace caraibe, INSEE Antilles-Guyane.

INSEE, 2006, Atlas des populations immigrées en Guyane, INSEE Antilles-Guyane.

Lézy Emmanuel, 2000, Guyane-Guyanes, une géographie sauvage de l'Orénoque à l'Amazone, Belin.

\section{NOTES}

1. Marché commun des pays de la Caraïbe.

2. Association des Etats de la Caraïbe.

3. Afrique Caraibes Pacifique, groupe de pays ayant signé des accords préférentiels avec l'Union européenne.

\section{RÉSUMÉS}

Sur la côte nord de l'Amérique du sud, la Guyane semble plutôt appartenir au monde caraïbe par son histoire, son peuplement et ses liens politico-économiques. Cependant, le haut niveau de vie découlant de son statut français et européen en fait un exutoire pour des dizaines de milliers d'immigrants originaires du Brésil voisin mais aussi, de plus en plus, du Pérou, amenant une prise de conscience par les autorités tant brésiliennes que guyanaises et françaises de sa situation d'interface entre Caraïbes, Amérique du sud et Europe. La récente admission de la France comme membre observateur du Traité de Coopération amazonien pour la Guyane montre le nouvel intérêt que suscite chez ses voisins cette terre française d'Amérique. Mais statut politique, écarts socio-économiques et mentalités freinent une plus grande intégration dans son environnement géographique. 
On the north coast of South America, French Guiana is appearing more as a caribbean territory because his history, his historical culture and his political and economical relations. But the high standard of living allowed by the french and european statute is transforming it as an outlet for ten of thousands of immigrants natives of the bordering Brazil and more and more Peru, inducing a sudden awareness of his situation of interface between the Caribbean world, South America and Europa. The recent admission of France as observer in the Organization of the Amazonian Cooperation Treaty owing to French Guiana is proving the new interest for this french country in America. But political statute, social and economical differences and mentalities are obstructing a greater integration in his geographical surroundings.

INDEX

Keywords : Brazil, Caribbean world, French Guiana, frontier cooperation, international migration, regional integration, South America

Mots-clés : Amérique du Sud, Brésil, Caraïbe, coopération transfrontalière, Guyane, intégration régionale, migration internationale

\section{AUTEUR}

\section{STÉPHANE GRANGER}

Stéphane Granger (granger.stephane@wanadoo.fr) est doctorant à l'IHEAL, Université de Paris III et enseignant en histoire-géographie et chargé de cours à l'Institut d'Etudes supérieures de la Guyane, Cayenne 